\title{
Hybrid Blood Purification Therapy Based on Camel Nanoantibody Immunoadsorbent on Patients with Chronic Renal Failure in Nephrology Department
}

\author{
Tao Ma $\mathbb{D}^{1},{ }^{1}$ Li Wang, ${ }^{2}$ and Xiuyong $\mathrm{Li} \mathbb{D}^{1}$ \\ ${ }^{1}$ Department of Blood Purification Center, Fuyang Second People's Hospital, Fuyang 236000, China \\ ${ }^{2}$ Department of Nephrology, Shenzhen Longhua District People's Hospital, Shenzhen, China \\ Correspondence should be addressed to Xiuyong Li; 1xy8726@126.com
}

Received 25 July 2020; Revised 27 October 2020; Accepted 11 November 2020; Published 17 December 2020

Academic Editor: Tifeng Jiao

Copyright (C) 2020 Tao Ma et al. This is an open access article distributed under the Creative Commons Attribution License, which permits unrestricted use, distribution, and reproduction in any medium, provided the original work is properly cited.

\begin{abstract}
This paper proposes a study on the effect of hybrid blood purification therapy based on camel nanoantibody immunoadsorbent on chronic renal failure patients in nephrology department. In this paper, the hybrid blood purification therapy based on camel nanoantibody immunoadsorbent was applied to patients with chronic renal failure, and the curative effect of the patients was studied. In order to highlight the effect, a control group was set up for comparison. In this paper, the clearance effect of small molecule toxin, middle molecular toxin, and dialysis adequacy was analyzed. This study found that the average dialysis adequacy of the control group was $1.23 \%$ and that of the experimental group was $1.26 \%$. The dialysis adequacy of the experimental group was significantly better than that of the control group. In addition, the clearance effect of small and medium molecular toxins in the experimental group was significantly better than that in the control group. It can be seen that the hybrid blood purification therapy based on camel nanoantibody immunoadsorbent is effective in the treatment of chronic renal failure patients in the department of nephrology. Therefore, it is of great value to apply camel nanoantibody in the clinical treatment of renal failure.
\end{abstract}

\section{Introduction}

Generally speaking, chronic renal failure refers to a variety of chronic kidney disease caused by the decline of glomerular filtration rate, resulting in toxic substances retention, electrolyte and acid base imbalance, endocrine disorders, and other clinical symptoms, and eventually develops into endstage renal failure. The incidence rate of chronic renal failure has shown an upward trend in recent ten years. The treatment of this disease is maintenance treatment. The process is long and requires a lot of manpower and financial resources, whether it is a heavy burden to the country or to the family, or to the individual. Therefore, it is very important to study the treatment of chronic renal failure.

Scholars have found that there is a natural heavy chain antibody in camels. This antibody does not contain light chain. It is a single domain antibody obtained after cloning the variable region of heavy chain antibodies in camel. This antibody is called nanoantibody and contains only one heavy chain variable region $[1,2]$. Camel-derived nanoantibodies have many characteristics, such as small molecular weight, strong stability, good solubility, easy expression, weak immunogenicity, strong penetration, strong targeting, and simple humanization. Because of these characteristics, camel-derived nanoantibodies have achieved good application effect in the diagnosis and treatment of diseases. The application of camel-derived nanoantibodies can also reduce the cost $[3,4]$. In this way, it will be of great significance to study the effect of camel-derived nanoantibodies in the treatment of renal failure patients, for the prevention and treatment of renal failure patients $[5,6]$.

Rabbit anti-camel antibodies can be detected by ELISA and western blotting, and their reactivity to all different camel IgG subclasses, which are purified from serum by differential affinity chromatography of protein-g and protein-a, is equal. Based on this, Shen used crude rabbit serum as raw material, 
purified by protein a column affinity chromatography, and prepared a standard stock of rabbit anti-camel antibodies $(1 \mathrm{mg} / \mathrm{ml})$ to detect camelid immunoglobulin and its derivative nanobodies $[7,8]$. On the other hand, rabbit anti-camel antibodies can also recognize nanobodies, and Shen provides an alternative method to detect the main body of these recombinant proteins $[9,10]$. His research discusses the synthesis of nanobodies, which has certain reference significance for the research of this article, but his research lacks the research on the clinical application of nanobodies [11, 12]. In order to explore the possibility of transforming immunotoxins into clinical antibodies, Cooper humanized the nanoantibody sequence and further truncated the pe38 toxin from Pseudomonas exotoxin $\mathrm{A}(\mathrm{PE})$ to produce a more protease resistant form $[3,13]$. Cooper successfully constructed the nanoantibody sequence-pe38 targeting CD7 molecule-modified immunotoxin and proved its potential in the treatment of CD7-positive malignant tumors, especially T-cell acute lymphoblastic leukemia $[14,15]$. Cooper's research lacks experimental data, and its persuasiveness is weak $[16,17]$. Ekart obtained high-titer anti-cd133 antibody from immunized camel, which provided basis for preparation of anti-cd133 nanoparticles [18, 19]. Ekart amplified the extracellular domain gene fragment of CD133 by PCR and connected it into pET28a plasmid to construct prokaryotic expression vector. The recombinant CD133 protein was induced to express by IPTG [20, 21]. In addition, he immunized one male Bactrian camel and two New Zealand rabbits with purified rcd133 antigen. The titer of polyclonal antibody and its specific binding to CD133 protein were detected by enzyme-linked immunosorbent assay (ELISA) and western blotting [22, 23]. Ekart's research confirmed that camel's high-titer anti-cd133 polyclonal antibody had higher specificity than rabbit's, which laid a good foundation for future experiments, but further exploration was needed in practical application [24].

Renal failure has always been a kind of disease that perplexes people's physical and mental health, and chronic renal failure is very serious to patients. With the development of science and technology, hybrid blood purification therapy based on camel nanoantibody immunoadsorbent has been applied in patients with chronic renal failure, but its efficacy needs further study. Therefore, this paper studies the effect of hybrid blood purification therapy based on camel nanoantibody immunoadsorbent on patients with chronic renal failure in nephrology department. In the research process, this paper analyzes the clearance effect of small molecular toxins, the clearance effect of medium molecular toxins, and dialysis adequacy. The results of this study show that the therapeutic effect of hybrid blood purification therapy based on camel nanoantibody immunoadsorbent is better than that of general blood purification therapy in all aspects. Therefore, it is of great value to apply camel nanoantibody in the clinical treatment of renal failure.

\section{Materials and Methods}

2.1. Materials. The main ingredients used in this paper are as follows: Sepharose CL-6B, DMSO, epichlorohydrin, 6aminohexanoic acid, ethanolamine, N, N-dimethylformamide, 1-ethyl-(3-dimethylaminopropyl) carbodiimide, hydrochloric acid, acetone, N-hydroxysuccinimide, phenolphthalein, sodium thiosulfate, immunoglobulin (Ig), human serum albumin (HSA), glycine, and camel-derived nanoantibodies.

The main instruments used are as follows: enzyme-labeled instrument, UV-1201 visible spectrophotometer, SHII circulating water vacuum pump, constant flow peristaltic pump BT0O-100M, glass column, and G3 sand core funnel.

\subsection{Experimental Methods}

2.2.1. General Information. Forty-five patients with chronic renal failure in the Department of Nephrology from December 2018 to December 2019 in a hospital were selected. The age of these patients was 70 years old, and the youngest was 21 years old. The average age was 46 years old, including 28 male patients and 17 female patients. These patients had no symptoms of infection and excluded complications such as tumor, heart failure, pulmonary edema and liver disease.

2.2.2. Experimental Design. Blood purification is mainly based on the principle of semipermeable membrane, the blood and dialysate are introduced into the instrument at the same time for penetration, and the flow direction is opposite. The purpose of removing metabolites or toxic substances in the body is achieved through dispersion, transportation, and adsorption on both sides of the hemodialysis membrane. On the other hand, the residual water in the body can be removed by excessive filtration and osmosis, and the useful components such as acid carbonate can be supplemented to correct the interference of electrolyte, acid, and alkali.

In the experiment, we include 45 patients, randomly divided into two groups, the experimental group and the control group, including 23 patients in the experimental group and 22 patients in the control group. In the two groups of patients, from the age level, there is no significant difference between the two groups, which is not statistically significant. On the other hand, from the gender point of view, the patients between the two groups were not statistically significant, that is, there was no significant difference between the two groups in gender. In the experimental process, the experimental group was treated with hybrid blood purification therapy based on camel nanoantibody immunoadsorbent, while the control group was treated with conventional blood purification therapy. Other variables were controlled under the same conditions to maintain a single variable and ensure the reliability of the data.

2.2.3. Observation Index. Before and after blood purification treatment, we need to draw up some observation indexes to determine the experimental effect. Therefore, we chose arterial blood sampling, then separated the serum, and carried out routine detection in the laboratory of the hospital to determine the serum creatinine (SCR), urea nitrogen (BUN), blood phosphorus (P), and other indicators of the two groups of patients. On the other hand, we also need to 
measure parathyroid hormone (PTH), which was completed by radioimmunoassay. In addition, we also need to calculate the clearance rate of each toxin and dialysis adequacy $(\mathrm{kt} / \mathrm{V})$ :

$$
\mathrm{SRR}=\left[\frac{(\mathrm{CB}-\mathrm{CA})}{\mathrm{CB}}\right] \times 100 \% .
$$

In formula (1), $\mathrm{CB}$ and $\mathrm{CA}$ are blood concentrations before and after dialysis.

Then, the calculation formula of dialysis adequacy $(\mathrm{kt} / V)$ is as follows:

$$
\left(\frac{\mathrm{Kt}}{V}\right)=-\operatorname{Ln}(R-0.008 t)+(4-3.5 R) \times\left(\frac{\mathrm{UF}}{W}\right) .
$$

In formula (2), $L n$ represents the natural logarithm, $R$ represents the ratio of bun concentration after dialysis to that before dialysis, UF represents ultrafiltration volume, and $W$ represents the weight of patients after dialysis.

2.2.4. Data Processing. In order to make the data more convincing, this paper carries out data processing. In the process of data processing, the data processing software is SPSS 20.0. On the other hand, the data in this paper were all tested by $F$-test and chi-square test. Among the data of each group, $p<0.05$ is the data with difference, that is, when the data of each group is $p<0.05$, the data will have statistical significance.

\section{Nanoantibody}

3.1. Structure of Nanoantibodies. As we know, antibodies are immunoglobulins secreted by $B$ lymphocytes or memory cells, which can specifically connect with the corresponding antigens and are an important part of the human immune system. Nanoparticles are the smallest known antigenic fragments. In general, the molecular weight of $s c F v$ is about $30 \mathrm{kd}$, while the molecular weight of nanoparticles is about $15 \mathrm{kD}$, which is equivalent to one-tenth of the normal antibody. On the other hand, there are sulfite bonds in the interior of the nanoparticles, and there are many hydrophilic residues on the surface. As we know, ordinary antibodies have six CDRs, while nanoantibodies only have three. However, although nanoantibodies have only three CDRs, they already have a complete antigen binding fragment. Not only that but also they have strong specificity and high affinity. We already know that nanoantibodies have three CDRs. How many CDRs does it have? It's four. The amino acid sequence of the skeleton region of the nanoantibody is relatively conservative. In this way, the amino acid sequence height of CDR can be changed, which is one of the determinants of the specificity of the nanoantibody.

\subsection{Characteristics of Nanoantibodies}

3.2.1. Small Molecule, Strong Structural Stability. The nanoparticles are very small and have a molecular weight of one-tenth of the normal antibody. At room temperature, nanoantibodies are easier to store and use than other ordinary antibodies. Moreover, at $37^{\circ} \mathrm{C}$ for a week, the nanoantibodies can maintain more than $80 \%$ of biological activity. On the other hand, the structure of the nanoantibody is very stable. Even if it is stored in the environment of $90^{\circ} \mathrm{C}$ for a long time, its nature can be restored, and its biological activity can be regained after the room temperature is restored.

\subsubsection{Good Solubility and Strong Tissue Penetration.} Hydrophobic residues and hydrophilic residues are related to the solubility of substances. In nanoantibodies, the hydrophobic residues become hydrophilic residues. In this way, the solubility of nanoantibodies is very good, which is better than that of general antibodies. On the one hand, nanoparticles have strong tissue penetration ability, can enter into dense tissue, play a role in it, and even effectively penetrate the blood-brain barrier.

3.2.3. High Affinity. Because of the structural specificity of nanoantibodies, nanoantibodies can reach the parts of the body that cannot be reached by normal antibodies. In this way, nanoantibodies can recognize specific epitope structures. In this way, both proteins and viruses in macromolecules, haptens, and peptides in small molecules can be recognized one by one with high affinity.

3.2.4. Weak Immunogenicity to Human Body. As we all know, the size and chemical structure of antibody molecules will affect immunogenicity, while the molecular weight of nanobodies is very small. Not only that but nanobodies have only one domain, so the immunogenicity of nanobodies will be weaker.

3.2.5. Easy to Express, Purify, and Modify. Nanobodies are not only small in molecular weight but also relatively simple in structure. Not only that but nanobodies are encoded by a single gene. In this way, it can be synthesized in microorganisms, and genetic engineering methods can be used to allow nanobodies to be expressed in large quantities in some microorganisms, thereby achieving large-scale production and obtaining more nanobodies, thereby reducing production costs.

\section{Camel-Derived Nanoantibody}

In order to maintain the normal function of heavy chain antibody, camel VHH gene has been selectively developed in many important functional sites. In the FR2 region of VH region of common antibodies, v37, g44, L45, and w47 interact with VL region, while in heavy chain antibody, these four amino acid residues are characteristic mutations, and the mutation sites are F (Y) 37, E44, R45, and g47. The changes of these four positions enhance the hydrophilicity of $\mathrm{VHH}$, so it is not necessary to form a bilateral structure with VL to keep the structure stable. VHH camel gene and human $\mathrm{VH}$ gene are very similar, the similarity is as high as $80 \%-90 \%$, and the structure is very similar. They contain three hypervariable regions (FVR) and four skeletal regions (FR). The difference 
between VHH camel and human VH is small, but cannot be ignored. The CDR1 and CDR3 of $\mathrm{VH}$ were longer than $\mathrm{VH}$. The CDR3 region of $\mathrm{VHH}$ gene is about 16-18 amino acids, while that of human and mouse $\mathrm{VH}$ gene is only 14 and 12 amino acids. At the same time, the $\mathrm{VHH}$ gene mutations of fr2v37, G44, L45, and w47 were significantly different.

4.1. Preparation of Adsorbent. The main synthetic steps of adsorbent are as follows:

\section{(1) Epoxy activation}

In this step, we first take Sepharose CL-6B of $5 \mathrm{~mL}$, then wash it with double distilled water and drain it, and then replace the ethanol in agarose gel with double distilled water. After full cleaning, Sepharose CL-6B is then dried, and $2 \mathrm{M} \mathrm{NaOH}$ is used to clean the gel, and then the liquid is drained. After this procedure is completed, we first put $5 \mathrm{~mL} 2 \mathrm{MNaOH}$ in the $50 \mathrm{~mL}$ cone bottle, then put the dried gel into the cone bottle, and shake it so that it can be mixed evenly. Then, $15 \mathrm{ml}$ DMSO and $2.5 \mathrm{ml}$ EPI were added. In addition, Sepharose CL-6B was placed in a $50 \mathrm{ml}$ conical flask and immediately placed in a constant temperature shaker at $37^{\circ} \mathrm{C}$. At the same time, timing was started. In this process, the reaction time was $12 \mathrm{~min}$. After the reaction, Sepharose CL-6B was cleaned with $30 \%$ acetone, $70 \%$ acetone, and $100 \%$ acetone. After that, Sepharose CL-6B was cleaned with $70 \%$ acetone and $30 \%$ acetone. After that, Sepharose CL-6B was cleaned with a large amount of water.

(2) Coupling 6-aminocaproic acid

In this step, Sepharose CL-6B-EPI gel is first placed in the funnel of the G3 sand core and then washed with $\mathrm{pH}=l 1$ concentration of $0.1 \mathrm{M}$ sodium carbonate/sodium bicarbonate buffer. After this step is completed, drain, and add $10 \mathrm{ml} \mathrm{pH}=l l, 0.1 \mathrm{M}$ sodium carbonate/sodium bicarbonate buffer solution. Finally, 6-aminocaproic acid with final concentration of $0.34 \mathrm{M}$ (about 250 times of epoxy density molar mass excess) was added, and the $\mathrm{pH}$ was adjusted with $2 \mathrm{~m} \mathrm{NaOH}$ to make $\mathrm{pH}=11,37^{\circ} \mathrm{C}$, and then the reaction was carried out at $170 \mathrm{rpm}$ for $17 \mathrm{H}$.

(3) Ethanolamine blocks epoxy groups

We put the dried Sepharose CL-6B into a dry conical flask and then add $20 \mathrm{ml}$ of $6 \%$ ethanolamine aqueous solution with $\mathrm{pH}=10$. The reaction was carried out at a temperature of $30^{\circ} \mathrm{C}$ and a rotation speed of $175 \mathrm{rpm}$ for at least 4 hours. After the reaction is finished, according to the corresponding sequence, wash with large amount of double steam water, wash with $1 \mathrm{M} \mathrm{NaCl}$, wash with double steam water, and then measure the density of remaining epoxy group.

(4) NHS

In this step, we first use agarose gel to wash with double distilled water. This cleaning requires a lot of double distilled water. After this procedure is finished, we use $1 \mathrm{M} \mathrm{NaCl}$ to wash and then use double distilled water to wash. After draining, we use DMF for flushing, which needs to be done twice. After these steps, we added $5 \mathrm{ml}$ DMF into a dry conical flask and added ED-CHCI with final concentration of $0.2 \mathrm{~m}$ and NHS of $0.2 \mathrm{~m}$, all of which were molar mass excess of 100 times epoxy density. After that, the reaction was carried out at a temperature of $30^{\circ} \mathrm{C}$ and a speed of $175 \mathrm{rpm}$ for $6 \mathrm{~h}$. After the reaction, it was cleaned with a large amount of DMF and stored in DMF at room temperature.

(5) Conjugation of nanoantibodies

In this step, we used ultrafiltration to change the solution into PBS buffer solution with $\mathrm{pH} 7.4$ and concentration of $10 \mathrm{~mm}$ and then determined the protein concentration by BCA method. After that, the agarose gel was washed with a large amount of water, and Sepharose CL-6B was added to the PBS buffer to wash and dry. Then, the $3.4 \mathrm{~mL}$ nanoantibody solution was added into the $10 \mathrm{~mL}$ centrifuge, and $1 \mathrm{~mL}$ Sepharose CL-6B gel was added to the centrifuge tube at a temperature of 30 degrees $\mathrm{C}$ for $175 \mathrm{rpm}$. The reaction time was $2 \mathrm{H}$. After centrifugation, the supernatant was taken for determination, and the protein content in the supernatant was determined, and the supernatant was taken out for ultrafiltration preservation. After that, it was washed with $1 \mathrm{~m} \mathrm{NaCl}$ for twice, and then Sepharose CL-6B was centrifuged at $8000 \mathrm{rpm}$. After centrifugation, the supernatant was collected and ultrafiltrated to recover the camel-derived nanoantibody. After that, the unreacted camel-derived nanoantibody in the supernatant was sucked away with a pipette gun. The remaining unreacted NHS was sealed with $6 \%$ ethanolamine solution of $\mathrm{pH} 8.0$ and centrifuged at $30^{\circ} \mathrm{C}$ and $175 \mathrm{rpm}$ for $4 \mathrm{~h}$.

(6) Preservation

After the above steps are completed, we repeatedly clean Sepharose CL-6B-camel nanoantibody with double distilled water and then wash it with PBS solution after being dried. After cleaning for two times, then drain again. After that, put the prepared adsorbent in $L \times \mathrm{PBS}$ with $\mathrm{pH} 7.4$, store it in a refrigerator at $4^{\circ} \mathrm{C}$, and then add the final concentration of $0.2 \%$ according to the volume of PBS sodium azide was used as antibacterial agent.

\section{Results and Discussion}

5.1. Analysis of Clearance Effect of Two Groups of Small Molecule Toxins. After 6 months, the clearance effect of small molecule toxins in the two groups was analyzed and compared with that before the experiment $[25,26]$.

5.1.1. Analysis of Clearance Effect of Blood SCR. The clearance effect of blood SCR in the two groups before and after the experiment was analyzed, and the results are shown in Figure 1. 


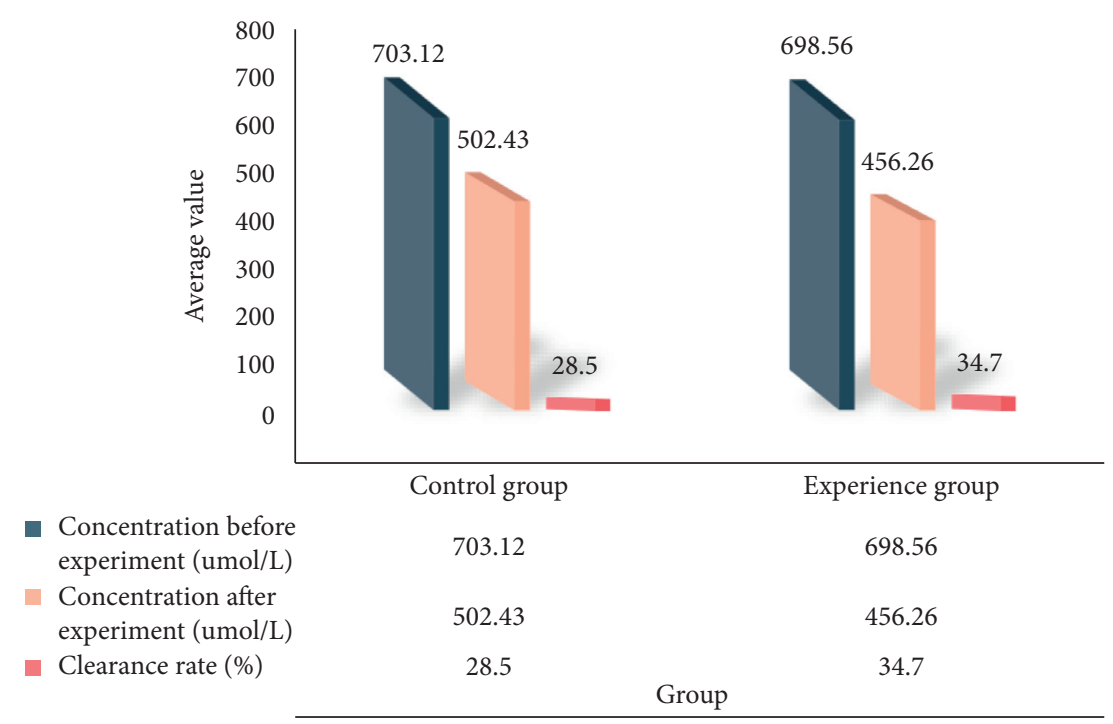

FIGURE 1: Analysis of SCR clearance effect in two groups.

Figure 1 shows the clearance effect of blood SCR between the two groups. In Figure 1, we can see that there is no significant difference between the two groups in blood SCR average concentration before the experiment. Among them, the average blood SCR concentration of the control group before the experiment is $703.12 \mu \mathrm{mol} / \mathrm{L}$, and the average SCR concentration of the experimental group is $698.56 \mu \mathrm{mol} / \mathrm{L}$, with no significant difference. After the experiment, the average value of blood SCR concentration in the control group was $502.43 \mu \mathrm{mol} / \mathrm{L}$, and the average value of blood SCR concentration in the experimental group was $456.26 \mu \mathrm{mol} / \mathrm{L}$. The average value of blood SCR concentration in the experimental group was significantly lower than that in the control group, and the data between the two groups had obvious differences. In addition, the average SCR clearance rate of the control group was $28.5 \%$ and that of the experimental group was $34.7 \%$. The average SCR clearance rate of the experimental group was significantly higher than that of the control group [27]. Therefore, the clearance of SCR in the experimental group was better than that in the control group.

5.1.2. Analysis of Clearance Effect of Blood P. The clearance effect of blood $p$ in the two groups before and after the experiment was analyzed, and the results are shown in Figure 2.

It can be seen from Figure 2 that, before the experiment, the average blood $P$ concentration of the control group was $2.43 \mathrm{mmol} / L$, and the average blood $P$ concentration of the experimental group was $2.56 \mathrm{mmol} / \mathrm{L}$. Therefore, there was no significant difference between the two groups before the experiment. Then, after the experiment, the average blood $P$ concentration in the control group was $1.98 \mathrm{mmol} / \mathrm{l}$, and the average blood $P$ concentration in the experimental group was $1.82 \mathrm{mmol} / \mathrm{l}$. The average blood $P$ concentration in the experimental group was lower than that in the control group. The blood $p$ clearance rate was calculated. The average

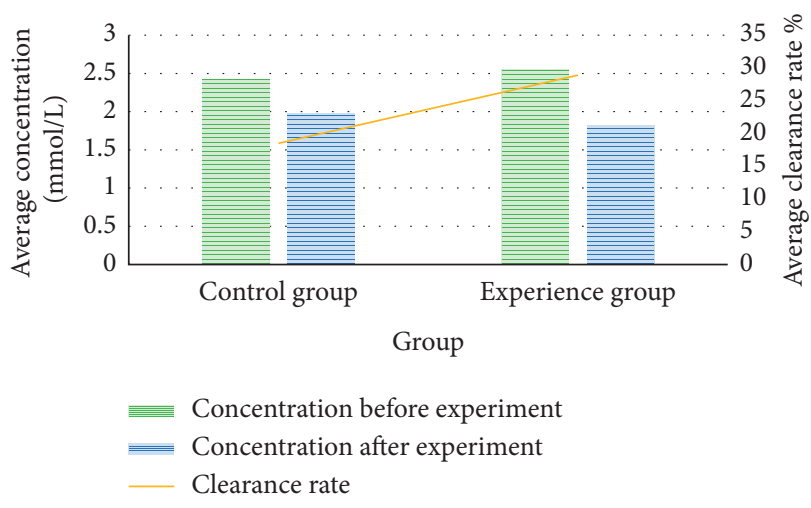

Figure 2: Analysis of clearance effect of $P$.

clearance rate of blood $p$ in the control group was $18.5 \%$, and the average clearance rate of blood $p$ in the experimental group was $28.9 \%$.

5.2. Analysis of Clearance Effect of Intermediate Molecular Toxin (PTH) between the Two Groups. The clearance effect of PTH between the two groups was analyzed. The results are shown in Table 1 and Figure 3.

It can be seen from Table 1 and Figure 3 that, before the experiment, there was no significant difference in blood PTH concentration between the two groups. The average blood PTH concentration of the control group was $296.35 \mathrm{pg} / \mathrm{ml}$ and that of the experimental group was $295.35 \mathrm{pg} / \mathrm{ml}$. However, after different treatment methods, the average value of PTH concentration in the control group was $248.56 \mathrm{pg} / \mathrm{ml}$, and the average value of PTH concentration in the experimental group was $125.35 \mathrm{pg} / \mathrm{ml}$. The average value of PTH concentration in the experimental group was significantly lower than that in the control group. In addition, the PTH clearance rate of the experimental group was $57.6 \%$, while that of the control group was only $16.1 \%$. It can be seen that the treatment effect of the experimental group is better. 
TABLE 1: Elimination effect of medium molecular toxin (PTH).

\begin{tabular}{lccc}
\hline Group & Concentration before experiment $(\mathrm{pg} / \mathrm{ml})$ & Concentration after experiment $(\mathrm{pg} / \mathrm{ml})$ & Clearance rate $(\%)$ \\
\hline Control group & $296.35 \pm 200.12$ & $248.56 \pm 100.12$ & 16.1 \\
Experience group & $295.36 \pm 231.35$ & $125.23 \pm 115.25$ & 57.6 \\
\hline
\end{tabular}

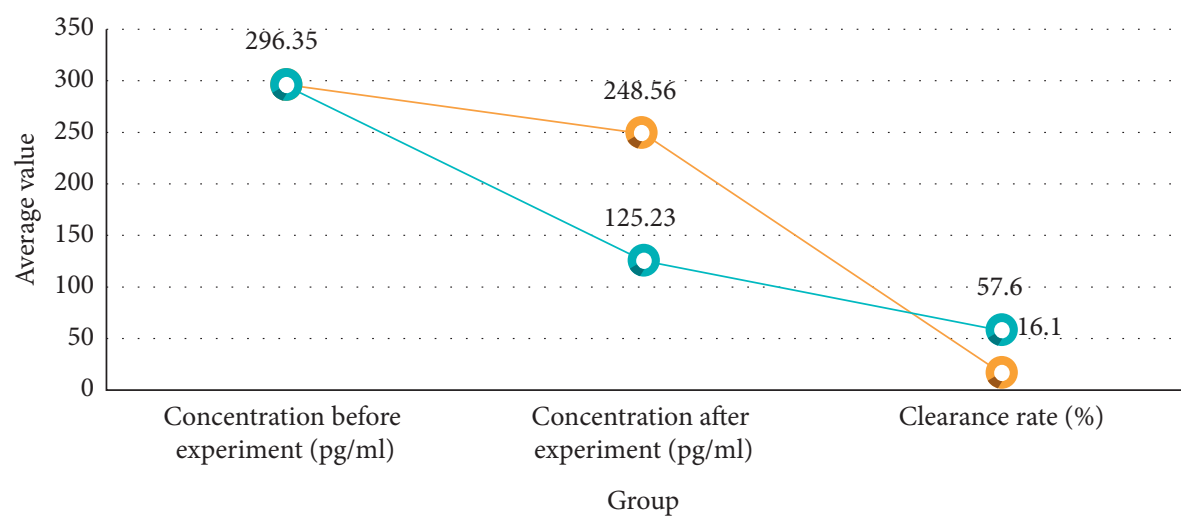

- - Control group

- Experience group

Figure 3: Analysis of the effect of removing middle molecular toxins.

TABLE 2: Analysis of blood bun concentration before and after the two groups.

\begin{tabular}{lcc}
\hline Group & Concentration before experiment $(\mathrm{mmol} / \mathrm{L})$ & Concentration after experiment $(\mathrm{mmol} / \mathrm{L})$ \\
\hline Control group & $26.80 \pm 11.19$ & $10.74 \pm 10.88$ \\
Experience group & $27.30 \pm 10.96$ & $9.50 \pm 12.50$ \\
\hline
\end{tabular}

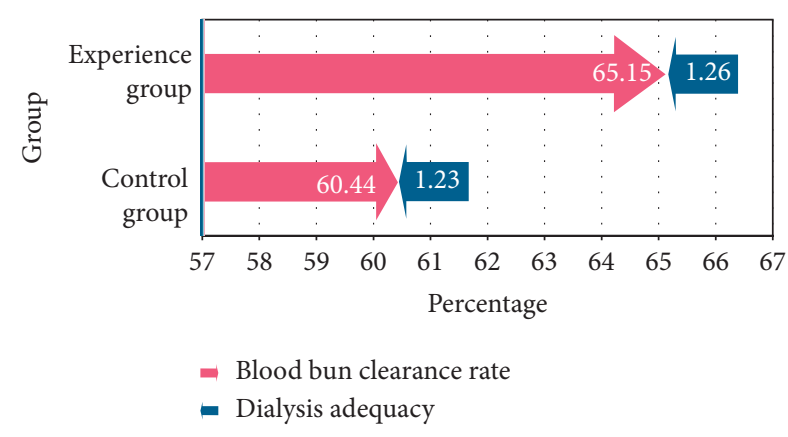

Figure 4: Comparison of blood bun clearance and dialysis adequacy between the two groups.

5.3. Dialysis Adequacy $(k t / V)$ Analysis between the Two Groups. The blood bun concentration between the two groups was analyzed. The results are shown in Table 2.

It can be seen from Table 2 that, before the experiment, there was little difference in blood bun concentration between the two groups, but after the experiment, the blood bun concentration between the two groups changed, and the data between the two groups had difference.

The blood bun clearance rate and dialysis adequacy (kt/ $V$ ) were compared between the two groups, and the results are shown in Figure 4.

It can be seen from Figure 4 that the average blood bun clearance rate of the control group was $60.44 \%$, and that of the experimental group was $65.15 \%$. There was significant difference between the two groups. On the other hand, the average value of dialysis adequacy in the control group was $1.23 \%$, and that in the experimental group was $1.26 \%$. The dialysis adequacy of the experimental group was significantly better than that of the control group.

\section{Conclusions}

Due to the high cost of kidney transplantation, the difficulty in matching the kidney source and the high risk of surgery and the clinical treatment of patients with chronic renal failure is based on dialysis. Dialysis treatment can significantly improve the quality of life of patients and prolong survival time. With the development of science and technology, hybrid blood purification therapy based on camelderived nanobody immunosorbent has emerged and has been proven effective through research. Many clinics have applied it to the treatment of patients with chronic renal failure in the Department of Nephrology, but its efficacy still needs to be explored in depth. For this reason, this article has conducted a study.

In the research process, this paper briefly introduces the preparation method of camel-derived nanoantibody immunoadsorbent and introduces the camel-derived nanoantibody to a certain extent. In the experiment, the control group was divided into two groups. The control group was treated with general blood purification therapy, 
and the experimental group was treated with hybrid blood purification therapy based on camel-derived nanoantibody immunoadsorbent. Then, the clearance effect of small molecule toxin, middle molecular toxin, and so on was used in the experimental group. In addition to the effect and dialysis adequacy analysis, the treatment effect of the two groups was compared.

In this study, we found that the hybrid blood purification therapy based on camel nanoantibody immunoadsorbent has better effect than the general blood purification therapy in the clearance effect of small and medium molecular toxins. In addition, the dialysis adequacy is also significantly better than that of the general blood purification chemotherapy method. Moreover, the data between the two groups have obvious differences. Therefore, the hybrid blood purification therapy based on camel nanoantibody immunoadsorbent has a good effect in the treatment of patients with chronic renal failure in the Department of Nephrology.

\section{Data Availability}

No data were used to support this study.

\section{Conflicts of Interest}

The authors declare no conflicts of interest.

\section{Authors' Contributions}

All authors have read and approved the final version of the manuscript.

\section{References}

[1] H. Muhammad, "Purification of polyclonal IgG specific for Camelid's antibodies and their recombinant nanobodies," Central European Journal of Biology, vol. 11, no. 1, pp. 1-9, 2016.

[2] Y. Yu, J. Li, X. Zhu et al., "Humanized CD7 nanobody-based immunotoxins exhibit promising anti-T-cell acute lymphoblastic leukemia potential," International Journal of Nanomedicine, vol. 12, pp. 1969-1983, 2017.

[3] D. Tsering and Q. Qian, "Electrolyte and acid-base disorders in chronic kidney disease and end-stage kidney failure," Blood Purification, vol. 43, no. 1-3, pp. 179-188, 2017.

[4] N. A. Bazaev, V. M. Grinval'd, K. V. Pozhar, and B. M. Putrya, "Mathematical model of a biotechnical system for extrarenal blood purification using a portable Artificial kidney apparatus," Biomedical Engineering, vol. 49, no. 5, pp. 322-326, 2016.

[5] A. Wieczorek, L. Matusiak, and J. C. Szepietowski, "Acquired perforating dermatosis associated with end-stage diabetic kidney failure in a hemodialysis patient," Iranian Journal of Kidney Diseases, vol. 10, no. 3, pp. 164-167, 2016.

[6] K. Legrand, B. E. Stengel, W. Ngueyon Sime et al., "Perceived health and quality of life in patients with CKD, including those with kidney failure: findings from national surveys in France," American Journal of Kidney Diseases, vol. 75, no. 6, pp. 868-878, 2020.

[7] J.-Q. Shen, L.-Y. Miao, and J.-F. Liu, "Fibroblast growth factor 23 resistance is probably involved in the mechanism of type 2 diabetes-induced nephropathy," Journal of Diabetes and Its Complications, vol. 30, no. 6, p. 1204, 2016.

[8] B. Ellen, "Chronic kidney disease: exploring value-based healthcare as a potential viable solution," Blood Purification, vol. 47, no. 1-3, pp. 156-165, 2019.

[9] H. F. Anja, "Perioperative hemodynamic instability and fluid overload are associated with increasing acute kidney injury severity and worse outcome after cardiac surgery," Blood Purif, vol. 43, no. 4, pp. 298-308, 2017.

[10] A. Okuyucu, "The restorative effect of ascorbic acid on liver injury induced by asymmetric dimethylarginine," Turkish Journal of Biology, vol. 40, pp. 1-10, 2016.

[11] B. S. Andrew, "Mineralocorticoid receptor antagonists in endstage renal disease: efficacy and safety," Blood Purification, vol. 41, no. 1-3, pp. 166-170, 2016.

[12] S. I. A. Hammour and K. Darwish, "Social problems resulting from chronic diseases (kidney failure and cancer): a survey study on the patients at Al-Bashear Hospital and Jordan Hospital," Dirasat Human \& Social Sciences, vol. 45, no. 1, pp. 183-201, 2018.

[13] D. S. Cooper, R. K. Basu, J. F. Price, S. L. Goldstein, and C. D. Krawczeski, "The kidney in critical cardiac disease," World Journal for Pediatric and Congenital Heart Surgery, vol. 7, no. 2, pp. 152-163, 2016.

[14] N. Fabio, "Robust kidney perfusion mapping in pediatric chronic kidney disease using single-shot 3D-GRASE ASL with optimized retrospective motion correction," Magnetic Resonance in Medicine, vol. 81, no. 5, pp. 2972-2984, 2019.

[15] J. Krauss and F. Botto, "Argentine consensus statement of cardiovascular risk evaluation in noncardiac surgery/brief version," Revista Argentina De Cardiología, vol. 84, no. 2, pp. 159-176, 2016.

[16] R. Sajad, "Relationship between circadian rhythm of blood pressure and renal failure severity in patients with chronic renal failure," Tehran University Medical Journal, vol. 75, no. 10, pp. 738-744, 2018.

[17] C. Y. Wang, "Cartilage MRI T2 relaxation time and perfusion changes of the knee in a 5/6 nephrectomy rat model of chronic kidney disease," Osteoarthritis \& Cartilage, vol. 25, no. 6, pp. 976-985, 2016.

[18] E. Robert, "Subendocardial viability ratio is impaired in highly proteinuric chronic kidney disease patients with low estimated glomerular filtration rate," Therapeutic Apheresis \& Dialysis, vol. 20, no. 3, pp. 281-285, 2016.

[19] O. V. Kamenskaia, A. S. Klinkova, and D. V. Khabarov, "Peripheral perfusion in CAD patients with various-stage chronic heart failure undergoing coronary artery bypass grafting," Angiol Sosud Khir, vol. 23, no. 1, pp. 67-73, 2017.

[20] K. Satoshi, "Effects of chronic kidney disease on myocardial washout rate of thallium-201 in patients with normal myocardial perfusion on single photon emission computed tomography," Annals of Nuclear Medicine, vol. 31, no. 9, pp. 703-708, 2017.

[21] K. Hirofumi, "Quantification of lung perfusion blood volume (lung PBV) by dual-energy CT in patients with chronic thromboembolic pulmonary hypertension (CTEPH) before and after balloon pulmonary angioplasty (BPA): preliminary results," European Journal of Radiology, vol. 85, no. 9, pp. 1607-1612, 2016.

[22] W. H. Reinhart, "The optimum hematocrit," Clinical Hemorheology and Microcirculation, vol. 64, no. 4, pp. 1-11, 2016.

[23] M. Aashish, B. K. Kobilka, and J. Steyaert, "Nanobodies to study G protein-coupled receptor structure and function," 
Annual Review of Pharmacology \& Toxicology, vol. 57, no. 1, pp. 19-37, 2017.

[24] M. J. Patel, "Cardiopulmonary exercise tests in patients with heart failure and chronic obstructive pulmonary disease," JACC: Heart Failure, vol. 4, no. 4, pp. 262-264, 2016.

[25] G. C. Zhang, J. Zhong, and Y. M. Yang, et al., Ternary BiVO4/ $\mathrm{NiS} / \mathrm{Au}$ nanocomposites with efficient charge separations for enhanced visible light photocatalytic performance," Chemical Engineering Journal, vol. 375, Article ID 122093, 2019.

[26] M. Feng, J. Gu, G. C. Zhang et al., "Homogeneous nickel bicarbonate nanocrystals as electrode materials for highperformance asymmetric supercapacitors," Journal of Solid State Chemistry, vol. 282, Article ID 121084, 2020.

[27] C. Jin, M. Feng, J. Zhong et al., "Insights into mechanism of size-controlled synthesis of $\mathrm{CH} 3 \mathrm{NH} 3 \mathrm{PbBr} 3$ perovskite quantum dots and large nanoparticles with tunable optical properties," Organic Electronics, vol. 82, Article ID 105712, 2020. 